\title{
Bose-Einstein source of intermittency in hadronic interactions
}

\author{
Tadeusz Wibig \\ Experimental Physics Dept., University of Łódź, \\ ul. Pomorska 149/153, PL-90-236 tódź, Poland
}

(September 16, 2018)

\begin{abstract}
The multi-particle Bose-Einstein correlations are the source of "intermittency" in high energy hadronic collisions. The power-law like increase of factorial moments with decreasing bin size was obtained by complete event weighing technique with gaussian approximation of space-time particle emitting source shape. The value of source size parameter was found to be higher than the common one fitted with the help of the standard Handbury BrownTwiss procedure.
\end{abstract}

24.60.Ky, 13.85.Hd, 12.40.-y

Typeset using REVTEX 
The use of intensity interferometry to determine space-time sizes of the particle emitting source is a well-established technique of high energy physics. The standard methods based on Handbury Brown-Twiss [1] (HBT) effect is to fit the Fourier transform of the source space-time density to the two-particle correlation function. The probability of finding one of the two emitted particles with the momentum $p_{1}$ and the second with $p_{2}$ is given by

$$
P_{\{12\}}=\int\left|\Psi\left(x_{1}, x_{2} ; p_{1}, p_{2}\right)\right|^{2} \rho\left(x_{1}\right) \rho\left(x_{2}\right) d^{4} x_{1} d^{4} x_{2},
$$

where $x_{1}$ and $x_{2}$ are the four-positions of the emission points each of them distributed in the "source" according to $\rho$. If the particles are bosons a symmetrization in the amplitude $\Psi$ evaluation leads to the well-known formula:

$$
P_{\{12\}} \sim 1+\left|\mathcal{F}_{12}\right|^{2}
$$

where the $\mathcal{F}_{12}$ can be related to the source distribution by

$$
\mathcal{F}_{12}=\int e^{\left(i q_{i j} x\right)} \rho(x) d^{4} x ; \quad q_{i j}=p_{i}-p_{j}
$$

There could be also other interpretations of $\mathcal{F}_{12}$. As for an example the one given in Ref. [2] derived on a basis of the relativistic string fragmentation picture.

The particular choice of the source space-time distribution (or, more general, the form of $\mathcal{F}_{12}$ ) leaves some degrees of freedom here, but the results do not depend very much of that choice. The most popular is the gaussian in space and exponential in time emission source shape. However for the present work we choose the form of $\mathcal{F}_{12}$ which is known as a gaussian parametrisation for its simplicity and because it is Lorentz-invariant:

$$
\mathcal{F}_{12}=e^{-\left(Q_{i j} R_{0}\right)^{2} / 2} ; \quad Q_{i j}^{2}=-\left(p_{i}-p_{j}\right)^{2}
$$

which leads to the well-known formula for the two-particle correlation function

$$
C_{2}\left(Q^{2}\right)=1+e^{-\left(Q R_{0}\right)^{2}}
$$

The $R_{0}$ can be still interpreted as a measure of space-time extension of the emitting source. 
The idea presented shortly above has extensively been used to analyze different high energy physics data since first work [3] by Goldhaber et al. Since that time many experimental and theoretical efforts have been made. The different source shapes were examined, some fine effects were predicted. Some difficulties were also found in the interpretation of the source shape while the source is moving very fast with respect to the laboratory system. However the main idea of HBT effect remains unchanged.

In the mid-eighties due to the work of Białas and Peschanski [4] a new interest for the particle correlation has arisen. The phenomenon called "intermittency" was found in the very small phase-space bin size analysis. Since the first measurements the experimentally available smallest bin size is reduced more than order of magnitude but, what is even more important, new techniques to study fine structures were developed. The "intermittency" of the particle creation process seen by Białas and Peschanski, which is in fact the fractal (selfscaled) behaviour of the multiparticle correlation measures at the very small phase-space scales, contradicts the standard Bose-Einstein statistics driven description given by Eqs. (2) and (3). The intermittent picture of hadronic creation was also inconsistent with existing models of particle production (like e.g. LUND hadronization model). The intermittent models like $\alpha$-model [4,5], geometrical branching model [6], one-dimensional model of intermittency by Dias de Deus [7] were invented but none of them achieved such a completeness and predictivity as high energy physics standards (LUND or DPM-type models). On the other hand, the treatment of the "intermittency" as a real new phenomenon was still not so obvious. In Ref. [8] different data sets were examined and as the last conclusion it is stated that the intermittency is caused by Bose-Einstein correlations in addition to a mechanism responsible for the power-law behaviour, in Ref. [9] authors claim that the observed "intermittent-like" behaviour of moments of multiplicity distributions can be understood as an effect driven by quantum statistical properties of the particle emitting system and it does not necessarily imply evidence for intermittency. The title of the Ref. [10] "Has intermittency been observed in multi-particle production?" is, to some extend, a good question still nowadays. 
As it has been said, the existing data gives a possibility to study intermittent (powerlow) behaviour of factorial moments in more than two decades wide phase-space distance measure (however it will be defined: rapidity, momentum or four-momentum difference, box volume etc.). It is clear now that the classical picture describing the HBT effect (Eq. (2)) is not valid. The factorial moment analysis were performed for $\mathrm{e}^{+} \mathrm{e}^{-}$annihilation into hadrons experiments, for hadronic collisions $\pi^{+} / \mathrm{K}^{+} \mathrm{p}$ at $\sqrt{\mathrm{s}}=22 \mathrm{GeV}$ and at much higher energy $\sqrt{\mathrm{s}}=630 \mathrm{GeV} p \overline{\mathrm{p}}$. There are also data from $p \mathcal{N}$ and $\mathcal{N} \mathcal{N}$ experiments. All they shows more or less definite a power-law like dependence on the bin size. However there is also very clear signal about the like and unlike charge difference of the correlation strength which suggests its Bose-Einstein origin. The possibility to achieve an agreement between those two, on the first sight contradicting, experimental facts will be discussed in the frame of common quantum physics.

It should be remembered that Eq. (2) was obtained in the case when only two particles were emitted from the source. That situation is of course different when one has got to do with multi-particle source [11]. In some particular cases (when there are really a small number of particles emitted in the large phase-space volume) the two-particle correlator given by Eq. (2) still can be used as at least a first approximation. But when one wants to look closely at the high multiplicity events or to study multiparticle correlations Eq. (2) has to be modified.

When $\mathrm{n}$ identical bosons are emitted the probability of the particular momenta configuration $\left\{\mathrm{p}_{\mathrm{i}}\right\}$ is given by:

$$
P_{\{n\}} \sim \sum_{\sigma} \mathcal{F}_{1 \sigma(1)} \mathcal{F}_{2 \sigma(2)} \ldots \mathcal{F}_{n \sigma(n)}
$$

where $\sigma$ is a permutation of a sequence $\{1,2, \ldots, n\}, \sigma(\mathrm{i})$ is the i-th element of this permutation and the sum is over all $n$ ! permutations.

To see what real difference is introduced by such complete treatment the two-particle correlation function like that in the Eq. (2) in the case of three particle emitting source is written explicit below: 


$$
P_{3} \sim 1+\left|\mathcal{F}_{12}\right|^{2}+\left|\mathcal{F}_{13}\right|^{2}+\left|\mathcal{F}_{23}\right|^{2}+2\left|\mathcal{F}_{12}\right|\left|\mathcal{F}_{13}\right|\left|\mathcal{F}_{23}\right| .
$$

If all three particles are very close to each other the statistical weight of such events tends to $6=\mathrm{n}$ !. The limit for two-particle correlator in n-particle emitting source is n! not 2 like it comes form Eq. (2). The same limit was obtained in Ref. [9] but it was interpreted as a limit of $\mathrm{n}$-th factorial moment. The multiplicity distribution in the very small phase-space bin tends to the geometrical one which, on the other side, can be treated as Bose-Einstein statistics driven multiplicity distribution while $\mathrm{n} \rightarrow \infty, \delta \rightarrow 0$ with $\mathrm{n} \delta=$ const.

Quite different approach to Bose-Einstein phenomenon is discussed in Ref. [12]. The authors argued for the local nature of the Bose-Einstein effect. In general, their treatments leads to the weighing procedure with the event probability proportional to:

$$
P_{\{n\}}^{\prime} \sim \sum_{\text {all pairs }}\left(1+\left|\mathcal{F}^{\prime}{ }_{i j}\right|^{2}\right) .
$$

The definition of $\mathcal{F}^{\prime}$ in Eq. (8) is not given by Eq. (四) but is based on string fragmentation picture. However the difference is rather in the physical interpretation than in the general behaviour. It should be noted that Eq. (8) overestimates the very close particle limit. It is there equal to $2^{\mathrm{n}(\mathrm{n}-1) / 2}$. The arguments for such a treatment are discussed in Ref. 112] (similar attempt is presented in Ref. [13]) and will not be discussed here. One of the arguments not given there but of the practical importance is that the above idea can be easily incorporated into the Monte-Carlo event generator. It was in fact done in the LUBOEI subroutine which is a part of LUND hadronization scheme JETSET 7.3. The general difference between the Ref. [12] strategy and proposed in the present paper is in the fact that sum in the Eq. (8) is performed over permutations of the particle ensemble in which only two particles are exchanged (locality of Bose-Einstein interaction) while in our treatment all event permutations can give a contribution to the event weight (global Bose-Einstein approach). The importance of many particle exchange contributions will be discussed later on.

The problem with complete weighing procedure is also a practical one. The sum over $n$ ! elements can be performed easily for about of ten particles or less. For higher multiplicities 
the calculation time rise tremendously. But it is quite clear than for the two very distant particle exchange the contribution coming from all permutations concerning that particular exchange is negligible. The algorithm was invented to omit all the negligible permutations and calculations of the weights according to Eq. (6) became possible also for larger multiplicities. In the present paper only the data from NA22 experiment will be analyzed. The mean charged particle multiplicity is of order of 8 and the largest like type boson multiplicity (in one chain, as will be discussed later) in the sample of about 500000 our Monte-Carlo generated events does not exceed 15 .

To study the influence of Bose-Einstein weighing method on the shape of the fluctuations in small bins the sample of events in the "world of absence of Bose-Einstein correlation" is needed. There is a number of Monte-Carlo generators which can be used to get this. In the present work the one called Geometrical Two-Chain was used. It is described in details in Refs. [14]. The advantage of that generator is the minimum of correlations introduced there. The ones existing are due to the conservation requirements (charge, barion number, strangeness, momentum and energy), the resonance production and the large scale clustering due to chain mass distribution in the model. There are also correlations connected with the hadronization procedure adapted: the transverse momentum is conserved locally in the fragmenting chains so the subsequent hadrons incline to have the negatively correlated momenta perpendicular to the interaction axis. Our chain fragmentation picture leads also to ordering in rapidity of subsequently produced hadrons. All that features are present in most of the models working on the partonic level. The last but very well seen specially for large bin sizes is a contribution related to non-poissonian multiplicity distribution in the multiparticle production.

The main interaction characteristics are very well reproducible by the generator as it was shown in Refs. [14].

About 500000 of non-single-diffractive events for $\pi^{+}$and $\mathrm{K}^{+}$interactions with proton at laboratory momentum of $250 \mathrm{GeV} / \mathrm{c}$ were generated and combined to get the reference sample without Bose-Einstein correlations included. Then for each event the weight was 
calculated according to Eqs. (4) and (6). In principle the Bose-Einstein weighing procedure could change the multiplicity distribution (what was one of the argument against global treatment of Bose-Einstein correlation in Ref. [12] ). To avoid this the weights were renormalized to get the average value of the weights for $n$ identical bosons equal to 1 and these were used afterward. The detail comparison with the experimental data leads to the conclusion that if the Bose-Einstein symmetrization were performed for the whole events then the correlations are too strong for very small bin sizes. In our model there is only one parameter to be adjusted, correlation radius $R_{0}$, while in the standard HBT procedure there is also the incoherence parameter which allows to make softer the correlation strength. In the Geometrical Two-Chain model particles are produced by the fragmentation of two well-defined chains so there is a natural subdivision of all secondary particles to two distinct classes. To make the correlation weaker there is a possibility to symmetrize amplitudes $\Psi$ not over all particle exchanges but only over the exchanges of the particles produced from the same chain.

The very convenient variable to study the two-particle correlation is the differential form of the second factorial moment as it was used in Ref. [15]. The definition using density integral method [16] is:

$$
D_{2}\left(Q^{2}\right)=\frac{1}{N o r m} 2 \sum_{i<j} \Theta\left(Q^{2}-Q_{i j}^{2}\right) \times \Theta\left(Q_{i j}^{2}-Q^{2}+\delta\right),
$$

were $\Theta$ is the Heaviside unit step-function and Norm is a normalization term defined by the so-called "mixed events" technique. The particles used for the normalization were chosen randomly from the all event "pool" of the large number of generated interactions, ensuring that they belongs to different real events. To avoid in the reference sample the correlations due to non-poissonian multiplicity distribution in hadronic interactions the multiplicity in the mixed events were taken from Poisson distribution with the average value the same as in M-C generated events.

In the particular NA22 experimental data which we want to compare with the rapidity cut $|\mathrm{y}|<2$ has been used. Thus in all the calculations the same cut is applied. In the 
experimental procedure there was also not possible, in general, to determined the particle masses so all the particles (except low energy proton and very energetic particles in $\mathrm{K}^{+}$ induced interactions $\mathrm{p}_{\mathrm{lab}}>150 \mathrm{GeV} / \mathrm{c}$ ) were treated as pion. The same procedure has been used in our analysis of the Monte-Carlo events. The experimental accuracy of particle four momentum difference determination described in Ref. [17] was taken into account in the calculations as well. The calculations od $D_{2}$ was performed for all charged particles as well as for like and unlike charge combinations. The results are presented in Fig. 11 by the solid line. The remained correlations produced in the Geometrical Two-Chains model, which were indicated above, leads to the outcome depicted by the dotted line. It represents the result of the correlation calculations without Bose-Einstein weighing.

It is seen that the power-low-like behaviour of $D_{2}\left(q^{2}\right)$ is quite well reproduced by our weighing method. The small overestimation of the unlike particle correlator at fourmomentum difference of about $Q^{2} \sim 10^{-2} \div 10^{-1}(\mathrm{GeV} / \mathrm{c})^{2}$ is a consequence of the strict ordering in rapidity of the chain fragmentation products which introduces always between close (in rapidity) like type charged hadron the one with the opposite sign. The fourmomentum difference of that unlike charged pairs is determined by transverse momentum distributions so the effect does not influence the very small bin size analysis.

However, the main argument for intermittency comes from the analysis of the higher multiplicity correlation measurements. To study this effects the correlation measures have to be defined for three- and more particle systems. The most commonly used variables are the factorial moments. For practical purposes the best method of factorial moment calculations is again the one proposed in Ref. [16] density integral method.

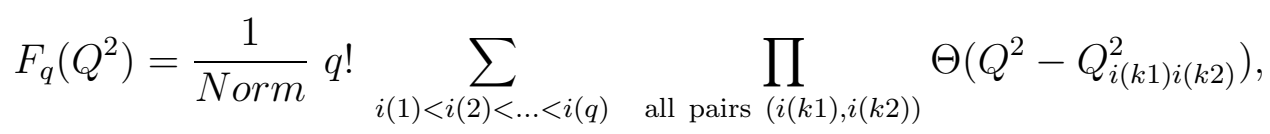

with the normalization by mixed event technique again. Results of our calculations are presented in Fig. 2. The power-law like increase of factorial moments with decreasing bin size is again quite well reproduced in the whole range of $Q^{2}$ measured experimentally.

In Figs. 1 and 2 the results of event weighing defined by Eqs. (4) and (6) with the sum 
over permutations with only one particle pair exchange are also presented (by the dashed line). As it is seen the effect (for the same value of $R_{0}$ parameter in Eq. (4)) ) is much weaker. This illustrate the importance of global treatment of Bose-Einstein correlation. The introduction to the sum of the weights very many relatively small terms leads to really great increase of the effect.

To reproduce the shape of $D_{2}\left(Q^{2}\right)$ and $F_{2}\left(Q^{2}\right)$ dependencies measured by NA22 experiment the value of the parameter $R_{0}$ in Eq. (雨) had to be adjusted. The large statistical fluctuations of the weights influence the estimation of source size parameter so the accuracy achieved is not higher that $10 \%$. In Ref. 15 the source size was found using the standard technique of HBT effect (Eq. (5) ). The value found there was $(0.82 \pm 0.02) \mathrm{fm}$. Our complete weighing procedure gives stronger correlations ( even after weights renormalization ) so the value of $R_{0}$ used to obtain the results given in Figs. 1 and 2 is about $50 \%$ higher what gives the source radius of about $1.25 \mathrm{fm}$ in the gaussian approximation (Eq. (4) ) interpretation.

To summarize, the importance of the global treatment of Bose-Einstein correlation has been shown. The symmetrization over all permutations leads to the power-low like behaviour of factorial moments in the four-momentum difference regions where they are measured experimentally. The more detail analysis is in progress and the results will be presented elsewhere. 


\section{REFERENCES}

[1] R. Handbury Brown, R. Q. Twiss, Philos Mag. 45, 663, (1976), Nature 178, 1046 (1956).

[2] B. Andersson and W. Hofmann, Phys. Lett. B 169, 364 (1986).

[3] G. Goldhaber et al., Phys. Rev. Lett. 3, 181, 1959, Phys. Rev. 120, 300 (1960).

[4] A. Białas and Peschanski, Nucl. Phys. B 273, 703 (1986).

[5] A. Białas and Peschanski, Nucl. Phys. B 308, 857 (1988).

[6] R. Hwa and J. C. Pan, Phys. Rev. D 45, 106 (1992).

[7] J. Dias de Deus, Phys. Lett. 194, 297 (1987).

[8] M. Charlet, Yad. Fiz. 56, 79 (1993).

[9] P. Carruthers, E. M. Friedlander, C. C. Shih and R. M. Weiner, Phys. Lett. B 222, 487 (1989).

[10] A. Capella, K. Fiałkowski and A. Krzywicki, Phys. Lett. B 230, 149 (1989).

[11] W. A. Zajc, Phys. Rev. D 35, 3396 (1987).

[12] L. Lönnblad and T. Sjöstrand, Phys. Lett. B 351, 293 (1995).

[13] X. Artru and M. G. Bowler, Z. Phys. C 37, 293 (1988).

[14] T. Wibig and D. Sobczyńska, Phys. Rev. D 49, 2268 (1994), ibid. 50, 5657 (1994).

[15] N. M. Agababyan et al., Z. Phys. C 59, 405, (1993).

[16] P Carruthers, Ap. J. 380, 24 (1991), P. Lipa "On the measurement of correlations and intermittency, Arizona preprint AZPH-TH/91-47, 1991 (unpublished), P. Lipa, P Curruthers, H. C. Eggers and B. Buschbeck, Phys. Lett. B 285, 300 (1992), H. C. Eggers, P. Lipa, P. Carruthers and B. Buschbeck, Phys. Rev. D 48, 2040 (1993). 
[17] W. Kittel (EHS/NA22 Collaboration), "Density Fluctuations in NA22", University of Nijmengen preprint, HEN-364, 1993 (unpublished). 


\section{FIGURES}

FIG. 1. The differential second factorial moments for a) all charged, b) like-charged and c) unlike-charged pairs as a function of four-momentum difference. The data points are from NA22 experiment. Solid line represents the result of our complete Bose-Einstein weight method, dotted shows the correlations in used Geometrical Two-Chain model. Dashed line is for the sum in Eq. (6) over only one pair of boson exchanges.

FIG. 2. The factorial moments a) for negatives and b) for all charged particles as a function of four-momentum difference. The data points are from NA22 experiment. 